\title{
Galilean Generalized Robertson-Walker spacetimes: a new family of Galilean geometrical models
}

\author{
Daniel de la Fuente* and Rafael M. Rubio ${ }^{\dagger *}$ \\ * Departamento de Geometría y Topología, \\ Universidad de Granada, 18071 Granada, Spain \\ E-mails: delafuente@ugr.es \\ † Departamento de Matemáticas, \\ Universidad de Córdoba, 14071 Córdoba, Spain \\ E-mail: rmrubio@uco.es
}

\begin{abstract}
We introduce a new family of Galilean spacetimes, which are relevant in the context of a generalized Newton-Cartan Theory. We study its geometrical structure and analyse the completeness of its inextensible free falling observers. This sort of spacetimes constitutes the local geometric model of a much wider family of spacetimes admitting certain conformal symmetry. Moreover, we find some sufficient geometric conditions which guarantee a global splitting of a Galilean spacetime as a Galilean Generalized Robertson-Walker spacetime.
\end{abstract}

Keywords: Leibnizian and Galilean structures, generalized Robertson-Walker spacetime, irrotational conformal vector fields, geodesic completeness, global splitting theorems.

MSC 2010: 53Z05, 53C80, 53B50.

\section{Introduction}

General Relativity is so far the most accurate and successful theory to describe the space-time structure and the gravitational phenomena. The evolution of the universe on a large scale was aptly described in the first half of the 20th century by means of the Robertson-Walker cosmological models (or fairly, Friedmann-Lemaitre-Robertson-Walker models). These models assume that the matter distribution and the "space relative to the family of observers commovil with the matter" are homogeneous and isotropic. These hypothesis may be weakened in order to describe a universe in a more accurate scale. With this objective, much more recently, new cosmological models have been introduced, as the Generalized Robertson-Walker (GRW) spacetimes [2]. This kind of relativistic spacetimes have been intensively studied from a mathematical perspective (see, for instance, [14], [15], [9], [11], [5].)

\footnotetext{
*The authors are partially supported Spanish MINECO and ERDF proyect MTM2016-78807-C2-1-P.
} 
However, the geometric formulation of the Newtonian's Gravitation, firstly postulated by E. Cartan [7],[8], after the appearance of the Einstein's General Relativity Theory, is still of interest and significant for several reasons.

On one hand, it formulates the classical Newtonian gravitation as a covariant theory and shows that certain results previously considered as characteristic or singular of the theory of Relativity are shared by the (geometric) gravitational Newton-Cartan Theory. In fact, the Newtonian gravity also arises as a consequence of the curvature of a connection in the spacetime, which does not come from any semi-Riemannian metric. Moreover, in the geometric formulation of Newtonian's Gravity Theory, the spacetime structure is dynamical in the sense that it participates in the unfolding of physics rather than being a fixed backdrop against which it unfolds.

On the other hand, it allows to establish from an accurate and intrinsic way the well known claim about the Newtonian theory of Gravitation is a certain "limit" of the theory of Relativity.

Another important question is that a geometric approach enables possible generalizations of Newtonian Theory, via the assumption of certain symmetries on Galilean spacetimes (see Section 2), which are the geometrical "arena" for the Newton-Cartan gravitation. So, in [12] the author studies the symmetry imposed on a Galilean spacetime by the cosmological principle, obtaining the Galilean model analogous to the relativistic Robertson-Walker spacetimes. The notion of symmetry is clearly basic in Physics. On a geometrical spacetime model, symmetry is usually based on the assumption of the existence of a one-parameter group of transformations generated by a Killing or, more generally, by a conformal vector field (see, $[17])$.

In this work, we introduce a new family of Galilean geometrical models, which generalize the non relativistic Robertson-Walker spacetimes, in the same way that GRW spacetimes generalized the Friedmann-Lemaitre-Robertson-Walker spacetimes: the Galilean Generalized Robertson-Walker (GGRW) spacetimes (Sect. 3). A GGRW spcetime possesses an infinitesimal symmetry given by the existence of a timelike irrotational conformally Leibnizian vector field. Several geometrical properties and physical interpretations for this family of spacetimes are given in Section 3, as the possible existence of singularities or the completeness of its free falling observers.

Section 9 is devoted to the study of Galilean spacetimes wich admits a timelike irrotational conformally Leibnizian vector field (ICL). We show that a ICL Galilean spacetime must be locally a GGRW spacetime (Sect. 4). Finally, Section 13 is devoted to face the following kind of splitting problems: under what geometrical assumptions an ICL spacetime globally decomposes as a GGRW spacetime.

\section{Set up}

Recall that a Leibnizian structure on a (non relativistic) spacetime $M^{1}$ is a pair $(\Omega, g)$ consisting of a differential 1-form $\Omega \in \Lambda^{1}(M)$, nowhere null $\left(\Omega_{p} \neq 0, \forall p \in M\right)$ and a positive definite Riemannian metric $g$ on its kernel. Specifically, let us denote by $\operatorname{An}(\Omega)=\{v \in$ $T M, \Omega(v)=0\}$ the smooth $n$-distribution induced on $M$ by $\Omega$. If we denote by $\Gamma(M)$ the set

\footnotetext{
${ }^{1} M$ is a smooth connected manifold of any dimension $m=n+1 \geq 2$.
} 
of smooth vector fields on $M$, we may construct the subset $\Gamma(\operatorname{An}(\Omega))=\left\{V \in \Gamma(T M) / V_{q} \in\right.$ $\operatorname{An}(\Omega), \forall p \in M\}$. So, the map

$$
g: \Gamma(\operatorname{An}(\Omega)) \times \Gamma(\operatorname{An}(\Omega)) \longrightarrow C^{\infty}(M),(V, W) \mapsto g(V, W),
$$

is smooth, bilinear, symmetric and positive definite. Hence, $M$ is endowed with a subRiemannian structure defined on the bundle $\operatorname{An}(\Omega)$, i.e., the annihilator of the degenerate metric $\Omega \otimes \Omega$ (see [3] and [4], for details).

Points of $M$ are usually called events. The Euclidean vector space $\left(\operatorname{An}\left(\Omega_{p}\right), g_{p}\right)$ is called the absolute space at $p \in M$, and the linear form $\Omega_{p}$ is the absolute clock at $p$. A tangent vector $v \in T_{p} M$ is named spacelike if $\Omega_{p}(v)=0$ and, otherwise, timelike. Additionally, if $\Omega_{p}(v)>0\left(\right.$ resp. $\left.\Omega_{p}(v)<0\right), v$ points out the future (resp. the past).

An observer in a Leibnizian spacetime $M$ is a timelike future unit smooth curve $\gamma: J \longrightarrow$ $M$, i.e., its velocity $\gamma^{\prime}$ satisfies that $\Omega\left(\gamma^{\prime}(s)\right)=1$ for all $s \in J$. The parameter $s$ is called the proper time of the observer $\gamma$. A vector field $Z \in \Gamma(T M)$ with $\Omega(Z)=1$ is called a field of observers, this is, its integral curves are observers.

When the smooth distribution $\operatorname{An}(\Omega)$ is integrable (equivalently, if the absolute clock $\Omega$ satisfies $\Omega \wedge d \Omega=0$ ), the Leibnizian spacetime $(M, \Omega, g)$ is said to be locally sincronizable, and making use of the Frobenious Theorem (see [16]), it may be foliated by a family of spacelike hypersurfaces $\left\{\mathcal{F}_{\lambda}\right\}$. In this case, it is well-known that each $p \in M$ has a neighbourhood where $\Omega=f d t$, for certain smooth functions $f>0, t$, and the hypersurfaces $\{t=$ constant $\}$ locally coincides with a leaf of the foliation $\mathcal{F}$. Thus, any observer may be synchronized through the "compromise time" $t$, obtained rescaling its proper time. In the more restrictive case $d \Omega=0$, then the Leibnizian spacetime $(M, \Omega, g)$ is called proper time locally synchronizable, and one has, locally, $\Omega=d t$. Now, observers are synchronized directly by its proper time (up to a constant). When $\Omega$ is exact, $\Omega=d t$ for some function $t \in C^{\infty}(M)$, called the absolute time function. In this case, any observer may be assumed to be parametrized with $t$. Notice that the notion of (local and global) synchronizability is intrinsic to the Leibnizian structure, applicable for every observer, in contrast to the relativistic setting, where the analogous concepts have meanings only for fields of observers.

According to [4], a field of observers is called Leibnizian if the stages $\Phi_{s}$ of its local flows are Leibnizian diffeoemorphisms, that is, they preserve the absolute clock and space, i.e.,

$$
\Phi_{s}^{*} \Omega=\Omega, \quad \text { and } \quad \Phi_{s}^{*} g=g .
$$

On the other hand, the inertia principle must be codified through a connection on the spacetime. However, a Leibnizian structure has not a canonical affine connection associated. Then, it is required to introduce a compatible connection with the absolute clock $\Omega$ and the space metric $g$, i.e., a connection $\nabla$ such that

(a) $\nabla \Omega=0$ (equivalently, $\Omega\left(\nabla_{X} Y\right)=X(\Omega(Y))$ for any $X, Y \in \Gamma(T M)$ ).

(b) $\nabla g=0$ (i.e., $Z(g(V, W))=g\left(\nabla_{Z} V, W\right)+g\left(\nabla_{Z} W, V\right)$ for any $Z \in \Gamma(T M)$ and $V, W$ spacelike vector fields).

Such a connection is named Galilean. A Galilean spacetime $(M, \Omega, g, \nabla)$ is a Leibnizian spacetime endowed with a Galilean connection $\nabla$. In addition, $\nabla$ is said symmetric if its 
torsion vanishes identically $\left(\operatorname{Tor}_{\nabla}(X, Y)=\nabla_{X} Y-\nabla_{Y} X-[X, Y] \equiv 0\right)$. From a physical point of view, a symmetric connection is desirable since is completely determined by its geodesics, i.e., by the free falling observers of $M$. From now on, we will only consider symmetric Galilean connections on the spacetime.

Given two Galilean spacetimes $(M, \Omega, g, \nabla)$ and $\left(M^{\prime}, \Omega^{\prime}, g^{\prime}, \nabla^{\prime}\right)$, a diffeomorphism $F$ : $M \longrightarrow M^{\prime}$ is said to be Galilean if $F^{*} \Omega^{\prime}=\Omega, F^{*} g^{\prime}=g$ and $F^{*} \nabla^{\prime}=\nabla$, i.e., $\nabla_{d F(X)}^{\prime} d F(Y)=$ $\nabla_{X} Y$.

For each fixed field of observers $Z$ on a Galilean spacetime $(M, \Omega, g, \nabla)$, the gravitational field induced by $\nabla$ in $Z$ is given by the spacelike vector field $\mathcal{G}=\nabla_{Z} Z$. The vorticity or Coriolis field of $Z$ is the 2 -form $\omega(Z)=\frac{1}{2} \operatorname{Rot}(Z)$, defined as

$$
\omega(Z)(V, W)=\frac{1}{2}\left(g\left(\nabla_{V} Z, W\right)-g\left(\nabla_{W} Z, V\right)\right) \quad \forall V, W \in \Gamma(\operatorname{An}(\Omega)) .
$$

The main result of [4, Th.5.27] claims that, for a fixed field of observers $Z$ on a Leibnizian spacetime $(M, \Omega, g)$ with $d \Omega=0$, the set of all symmetric Galilean connections is bijectively mapped onto $\left(\Gamma(T M), \Lambda^{2}(\operatorname{An}(\Omega))\right)$. Each symmetric Galilean connection $\nabla$ is mapped to $(\mathcal{G}(Z), \operatorname{Rot}(Z))$. Thus, the gravitational field and the vorticity of a field of observers determine a unique symmetric Galilean geometry of the spacetime.

Additionally, a Leibnizian field of observers $Z$ in a Galilean spacetime $(M, \Omega, g, \nabla)$ is named Galilean if it is affine for $\nabla$, that is, $L_{Z} \nabla=0$, where $L$ denotes the Lie derivative. Finally, a Galilean spacetime is said Newtonian if the (symmetric) connection $\nabla$ restricted to the spacelike vectors is flat, and it admits an irrotational Galilean field of observers. This kind of spacetimes has traditionally represented the classical (non relativistic) geometric model of gravity.

\section{Galilean Generalized Robertson-Walker spacetimes}

In this section we introduce a new family of Galilean geometric models, which are the classical version of the relativistic Generalized Robertson-Walker spacetimes defined in [2].

Definition 1 Let $I \subseteq \mathbb{R}$ be a real interval, $(F, h)$ a n-dimensional connected Riemannian manifold, and $f \in C^{\infty}(I)$ a smooth positive function on $I$. A Galilean spacetime $(M, \Omega, g, \nabla)$ is called Galilean Generalized Robertson-Walker spacetime (GGRW) if $M=I \times F, \Omega=d \pi_{I}$, $g$ is the restriction to the bundle $\operatorname{An}(\Omega)$ of the following (degenerate) metric on $M$,

$$
\bar{g}=\left(f \circ \pi_{I}\right)^{2} \pi_{F}^{*} h,
$$

where $\pi_{I}, \pi_{F}$ are the canonical projections onto the open interval I and the fiber $F$ respectively, and $\nabla$ is the only symmetric Galilean connection on $M$ such that

$$
\nabla_{\partial_{t}} \partial_{t}=0, \quad \text { and } \quad \operatorname{Rot} \partial_{t}=0,
$$

where $\partial_{t}=\partial / \partial t$ is the global coordinate vector field associated to $t:=\pi_{I}$.

The vector field $\partial_{t}$ defines a field of observers in $M\left(\Omega\left(\partial_{t}\right)=1\right)$, which we will call commovil observers, by the similarity with the relativistic Robertson-Walker spacetimes. Then, the conditions (2) in above definition mean that commovil observers are free falling and they do not rotate. 
Example 2 Let us consider a $G G R W$ with $I=\mathbb{R}$ and $F=\mathbb{R}^{n}$ endowed with the usual Euclidean metric. If $f(t)=$ constant, then the Galilean connection coincides with the standard flat connection of the affine space $\mathbb{R}^{n+1}$. In addition, the commovil observers satisfy the necessary conditions to assure the Newtonian character of this spacetime.

\subsection{Some physical interpretations}

From a physical viewpoint, a GGRW spacetime can model a universe (large-scale) composed by matter (galaxies) only subjected to the proper gravity action. Their trajectories are identified with the integral curves of the geodesic vector field $\partial_{t}$. The cosmological principle leads to a spatially homogeneous and isotropic universe ${ }^{2}$, in such a way that its evolution is only produced by means dilatations and contractions with scale factor $f(t)$ depending only on the absolute time $t$. This situation is modelled by a (non relativistic) Robertson-Walker spacetime, namely, a GGRW with fiber $F$ a Riemannian manifold with constant sectional curvature (see, [12]). However, although the hypothesis of spatial homogeneity and isotropy is reasonable as a first approximation of the large scale structure of the universe, it could not be appropriate when we consider a more accurate scale. This fact motives the introduction of this new family of Galilean spacetimes.

On the other hand, for each event $p \in M$, we may parametrize $I \times\{p\}$ by $\gamma_{p}(t)=(t, p)$, being $\gamma_{p}$ a commovil observer in $\partial_{t}$. Set $q \in M, q \neq p$. The absolute distance between two instantaneous observers $\gamma_{p}$ and $\gamma_{q}$ in $\mathcal{F}_{t}=\{t\} \times F$ is given by the Riemannian distance in $\mathcal{F}_{t}$. In particular, when $f$ has positive derivative, the "spaces $\mathcal{F}_{t}$ are expanding" (or, alternatively, the distance between commovil observers is increasing). Conversely, if $f^{\prime}<0$, the separation between commovil observers is decreasing. When $f(t)=1$ (or constant) the commovil observers are relatively statics.

Notice that this family of spacetimes allows us to describe many scenarios. For instance, if we consider $I=\mathbb{R}, N$ compact and $f(t)=\frac{1}{1+t^{2}}$, the commovil observers measure an expanding universe during the time interval $(-\infty, 0)$, and contracting for $t \in(0,+\infty)$. There is no physical singularities, but the distances between two arbitrary observers can be arbitrarily small for $|t|$ big enough.

But we can also find GGRW spacetimes with physical singularities. Indeed, consider $I=(-a, a)$ and $f(t)=\sqrt{a^{2}-t^{2}}, a>0$. Since $\lim _{t \rightarrow \pm a} f(t)=0$ and $\lim _{t \rightarrow \pm a} f^{\prime}(t)= \pm \infty$, we deduce that the model has a Big Bang singularity at $t=-a$ and a Big Crunch at $t=a$. The reader may compare this model with the spatially closed Friedmann models [13, Chap.2].

In a Newtonian spacetime, the Poisson equation relates the density of mass of the universe with its intrinsic geometry, in an analogous way that Einstein equations in the relativistic setting,

$$
\operatorname{Ric}=4 \pi G \varrho \Omega \otimes \Omega .
$$

Here, Ric is the Ricci tensor of $\nabla, G$ the universal gravitational constant, and $\varrho$ the density of mass (the only source of the gravitational field).

\footnotetext{
${ }^{2}$ A Leibnizian spacetime $(M, \Omega, g)$ (with integrable distribution $\operatorname{An}(\Omega)$ ) is said to be spatially homogeneous if for any $p, q \in M$ located in the same leaf of the foliation, there exists a Leibnizian diffeomorphism carrying $p$ into $q$. Analogously, $M$ is called spatially isotropic at an event $p \in M$ if, for any spacelike vectors $v, w \in T_{p} M$, there is a Leibnizian diffeomorphism $\psi$ such that $\left.d \psi\right|_{p}(v)=w$.
} 
If we admit that this equation is also true for the GGRW spacetimes, we get

(i) $(F, h)$ must be Ricci-flat.

(ii) $\operatorname{Ric}\left(\partial_{t}, \partial_{t}\right)=-n \frac{f^{\prime \prime}}{f}=4 \pi G \varrho .^{3}$

Notice that, from $(i i)$, we immediately deduce that density of mass $\varrho$ must be constant at each instant, and the scale factor $f$ must satisfy $f^{\prime \prime}(t) \leq 0$ for all $t \in I$.

Corollary 3 If in a RRGW spacetime the Poisson equation holds and $f$ is not constant, then $I \neq \mathbb{R}$. In fact,

(i) if there exists $t_{0} \in I$ such that $f^{\prime}\left(t_{0}\right)>0$, then $-\infty<\operatorname{Inf}(I)$,

(ii) if there exists $t_{0} \in I$ such that $f^{\prime}\left(t_{0}\right)<0$, then $\operatorname{Sup}(I)<\infty$.

Notice that this result implies that if a GGRW spacetime satisfies the above assumptions, then is not geodesically complete (the integral curves of $\partial_{t}$ cannot be complete, see the following subsection), i.e., a Big Bang or a Big Crunch happens. We remark that this consequence is identical for the relativistic GRW spacetimes (see, [13, Chap. 12]).

\subsection{Completeness of free falling observers in a GGRW spacetime}

We now proceed to analyze when the inextensible free falling trajectories in a GGRW spacetime are complete. Physically we are looking for geometric assumptions that guarantee that every free falling observers lives forever.

First, we obtain an analogous result to the geodesic normalization lemma in semi-Riemannian manifolds.

Lemma 4 Let $\gamma$ be a geodesic in a GGRW spacetime. Then, $\Omega\left(\gamma^{\prime}\right)$ is constant along the trajectory of $\gamma$.

Proof. Since $\nabla_{\gamma^{\prime}} \gamma^{\prime}=0$, using $(a)$ it is directly obtained that $0=\Omega\left(\nabla_{\gamma^{\prime}} \gamma^{\prime}\right)=\gamma^{\prime}\left(\Omega\left(\gamma^{\prime}\right)\right)$.

The relevant cases correspond with $\Omega\left(\gamma^{\prime}\right)=0$ or 1 . The first one $\left(\Omega\left(\gamma^{\prime}\right)=0\right)$ means that $\gamma$ is spacelike and contained in a leaf $\mathcal{F}_{t}$ of the foliation of $\Omega$. As $\nabla$ coincides with the LeviCivita connection of $\left(\mathcal{F}_{t}, f(t)^{2} h\right)$, the completeness of this kind of geodesics is equivalent to the geodesic completeness of $(F, h)$. Thus, from now on we will deal with free falling observers $\left(\gamma\right.$ geodesic with $\left.\Omega\left(\gamma^{\prime}\right)=1\right)$.

Theorem 5 A GGRW spacetime is geodesically complete if and only if $I=\mathbb{R}$ and the fiber $(F, h)$ is (geodesically) complete.

Proof. Let $\gamma(s)=(\theta(s), \sigma(s))$ be a free falling observer. Since $\Omega\left(\gamma^{\prime}\right)=1$, we can write

$$
\gamma(t)=(t, \sigma(t)), \quad \text { and } \quad \gamma^{\prime}=\partial_{t}+\overline{\sigma^{\prime}},
$$

where $\overline{\sigma^{\prime}}$ is the horizontal lift of $\sigma^{\prime}$ from $F$ to $M$. Consequently, using that $\partial_{t}$ is geodesic and $\left[\partial_{t}, \overline{\sigma^{\prime}}\right]=0$, we have

$$
0=\nabla_{\gamma^{\prime}} \gamma^{\prime}=\nabla_{\overline{\sigma^{\prime}}} \overline{\sigma^{\prime}}+\frac{2 f^{\prime}}{f} \overline{\sigma^{\prime}} .
$$

\footnotetext{
${ }^{3}$ To compute $\operatorname{Ric}\left(\partial_{t}, \partial_{t}\right)$, formula (8) of the following section has been used.
} 
Since $\nabla_{\overline{\sigma^{\prime}}} \overline{\sigma^{\prime}}=\overline{\nabla_{\sigma^{\prime}} \sigma^{\prime}}$, and taking into account that $F$ is homothetic to each leaf of $\mathcal{F}$ (and homothecies preserve the connections), the completeness of Eq.(4) is equivalent to the completeness of

$$
\nabla_{\sigma^{\prime}}^{h} \sigma^{\prime}+\frac{2 f^{\prime}}{f} \sigma^{\prime}=0
$$

where $\nabla^{h}$ is the Levi-Civita connection of $h$. As $f \in C^{\infty}(\mathbb{R})$, a straightforward application of [6, Th.2] gives the completeness of the inextensible solutions of the non-autonomous Eq.(5), and the proof is ended.

\section{Irrotational conformally Leibnizian spacetimes}

In this section we present a wider family of Galilean spacetimes which locally exhibit the structure of a GGRW spacetime. As a previous step, we introduce the concept of conformally Leibnizian field of observers, generalizing the well-known notion of Leibnizian observer.

Definition 6 Let $(M, \Omega, g)$ be a Leibnizian spacetime. A vector field $X$ is called spatially conformally Leibnizian vector field if the Lie derivative of the absolute space metric satisfies

$$
L_{X} g=2 \lambda g,
$$

for some smooth function $\lambda \in C^{\infty}(M)$. If $X$ additionally verifies

$$
L_{X} \Omega=2 \lambda \Omega,
$$

for the same conformal factor $\lambda$, then $X$ is named conformally Leibnizian vector field.

Note that a conformally Leibnizian vector field is Leibnizian if and only if the conformal factor $\lambda$ is identically zero [4].

Remark 7 Condition (6) may be also expressed as

$$
X(g(V, W))=\lambda g(V, W)+g([K, V], W)+g([K, W], V), \quad \forall V, W \in \Gamma(\operatorname{An}(\Omega)) .
$$

Analogously, assumption (7) is equivalent to

$$
d \Omega(X, Y)+Y(\Omega(X))=\lambda \Omega(Y), \quad \forall Y \in \Gamma(T M),
$$

and means that distribution $\operatorname{An}(\Omega)$ is invariant along the flow of vector field $X$. So, if this distribution is integrable, the flow of $X$ carries each leaf of the foliation to other one.

The following result shows that the GGRW spacetimes admit a timelike conformally Leibnizian vector field.

Proposition 8 Let $(M=I \times F, \Omega=d t, g, \nabla)$ be a GGRW spacetime with scale factor $f \in C^{\infty}(I)$. Then, the vector field $K:=\left(f \circ \pi_{I}\right) \partial_{t}$ is irrotational and conformally Leibnizian and, consequently, satisfies the identity

$$
\nabla_{X} K=\left(f^{\prime} \circ \pi_{I}\right) X, \quad \forall X \in \Gamma(T M) .
$$


Proof. Let $V, W$ be spacelike vector fields. A straightforward computation shows

$$
\operatorname{Rot}(K)(V, W)=\left(f \circ \pi_{I}\right) \operatorname{Rot}\left(\partial_{t}\right)(V, W)=0 .
$$

Consider a fixed point $\left(t_{0}, p\right) \in M$, and $v, w \in T_{p} F$. Hence,

$$
\left.L_{\partial_{t}} g\right|_{\left(t_{0}, p\right)}((0, v),(0, w))=\eta^{\prime}(0),
$$

where $\eta(s)=\left.g\right|_{\left(t_{0}+s, p\right)}\left(d \Phi_{s}(0, v), d \Phi_{s}(0, w)\right)$, and $\Phi_{s}: M \rightarrow M$ is the flow of the vector field $\partial_{t},(t, q) \mapsto(t+s, q)$. Thus, $d \Phi_{s}(0, v)=(0, v)$ for all $s \in \mathbb{R}$, and it follows

$$
\eta(s)=f^{2}\left(t_{0}+s\right) h(v, w) .
$$

As consequence, we have

$$
L_{\partial_{t}} g=\left(\frac{2 f^{\prime}}{f} \circ \pi_{I}\right) g
$$

Finally, taking into account that $V\left(f \circ \pi_{I}\right)=0$ for any spacelike vector field, it holds that

$$
L_{K} g=2\left(f^{\prime} \circ \pi_{I}\right) g
$$

and $K$ is spatially conformally Leibnizian with conformal factor $\lambda=f^{\prime} \circ \pi$.

From (9) and the irrotational character of $K$ we obtain

$$
g\left(\nabla_{V} K, W\right)=\left(f^{\prime} \circ \pi_{I}\right) g(V, W), \quad \forall V, W \in \Gamma(\operatorname{An}(\Omega)) .
$$

Now let us consider $X \in \Gamma(T M)$, which may be expressed at a point $p$ as

$$
\left.X\right|_{p}=\left.\varrho \partial_{t}\right|_{p}+\sum_{i} \mu_{i} v_{i}, \quad \mu_{i} \in \mathbb{R},
$$

where $\left\{v_{i}\right\}_{i}$ is an orthonormal basis of $\left(\operatorname{An}\left(\Omega_{p}\right), g_{p}\right)$. Therefore,

$$
\left.\nabla_{X} K\right|_{p}=\left.\Omega\left(\nabla_{X} K\right)_{p} \partial_{t}\right|_{p}+\sum_{i} g_{p}\left(\nabla_{X_{p}} K, v_{i}\right) v_{i}
$$

Making use of $\nabla_{\partial_{t}} \partial_{t}=0$, some computations give

$$
\left.\nabla_{X} K\right|_{p}=\left.X_{p}\left(f \circ \pi_{I}\right) \partial_{t}\right|_{p}+\left(f^{\prime} \circ \pi_{I}\right)(p) \sum_{i} \mu_{i} v_{i}=\left.\left(f^{\prime} \circ \pi_{I}\right) X\right|_{p},
$$

and we obtain (8). From this equality, the conformally Leibnizian character of $K$ is easily deduced.

Definition 9 Let $(M, \Omega, g, \nabla)$ be a Galilean spacetime, whose absolute clock is closed $(d \Omega=$ $0)$. If $M$ admits a timelike vector field $K \in \Gamma(T M)$ satisfying

$$
\nabla_{X} K=\rho X, \quad \forall X \in \Gamma(T M)
$$

$M$ is said Irrotational Conformally Leibnizian spacetime (ICL). 
Remark 10 Notice that condition (10) directly implies that $K$ is conformally Leibnizian and $\operatorname{Rot}(K)(V, W)=0$, for all spacelike vector fields $V, W$.

As a first consequence of definition 9 , we obtain that functions $\Omega(K)$ and $\rho$ are constant on each leaf of the foliation induced by $\Omega$.

Lemma 11 Let $(M, \Omega, g, \nabla)$ be a ICL spacetime with irrotational conformally Leibnizian vector field $K$ and conformal factor $\rho$. Then

$$
V(\Omega(K))=0 \quad \text { and } \quad V(\rho)=0, \quad \forall V \in \Gamma(\operatorname{An}(\Omega)) .
$$

Proof. From (10) and (a), we have that

$$
V(\Omega(K))=\Omega\left(\nabla_{K} K\right)=\rho \Omega(V)=0
$$

and $\rho=\frac{K(\Omega(K))}{\Omega(K)}$, for any spacelike vector field $V$. Finally, since $[V, K]$ is spacelike, we have

$$
V(\rho)=\frac{1}{\Omega(K)} K(V(\Omega(K))=0
$$

We have just seen that each GGRW is an ICL spacetime. Next theorem ensures that any ICL spacetime is locally a GGRW spacetime.

Theorem 12 Let $(M, \Omega, g, \nabla)$ be an ICL spacetime. For each $p \in M$, there exist an open neighbourhood of $p, \mathcal{U}$, and a Galilean diffeomorphism $\Psi: N \longrightarrow \mathcal{U}$, where $N$ is a GGRW spacetime.

Proof. Let $Z=\frac{1}{\Omega(K)} K$ be the field of observers associated to $K$, and let $\Phi_{s}$ be its (local) flow. Fixed a point $p \in M$, we take a neighbourhood $U_{p}$ in the leaf $\mathcal{F}_{p}$ of the foliation induced by $\Omega$ passing through $p$. Consider $I \in \mathbb{R}, 0 \in I$, a suitable interval such that the flow $\Phi_{s}$ is well-defined and one-one. Then, we may define the map

$$
\Psi: I \times U_{p} \longrightarrow M, \quad(s, q) \longmapsto \Phi_{s}(q),
$$

which satisfies,

$$
\left.d \Psi\right|_{(s, q)}(1,0)=\left.Z\right|_{\Phi_{s}(q)},\left.\quad d \Psi\right|_{(s, q)}(0, v)=\left.d \Phi_{s}\right|_{q}(v),
$$

for all $(s, q) \in I \times U_{p}$ and $v \in T_{p} U_{p}$. Now, identifying $\pi_{I} \equiv t$ and $\partial_{t} \equiv(1,0)$, and taking into account that the flow of $Z$ preserves $\Omega$, we have

$$
\Omega\left(d \Psi\left(\partial_{t}\right)\right)=\Omega(Z)=1=d t\left(\partial_{t}\right)
$$

and

$$
\Omega\left(\left.d \Psi\right|_{(s, q)}(0, v)\right)=\Omega\left(\left.d \Phi_{s}\right|_{q}(v)\right)=\left.\Phi_{s}^{*} \Omega\right|_{q}(v)=\Omega_{q}(v)=0 .
$$

As a consequence, $\Psi^{*} \Omega=d t$, and each level set of $t$ corresponds with certain open set $\mathcal{U} \cap \mathcal{F}_{a}$ of some leaf of the foliation $\mathcal{F}$ induced by $\Omega$. 
On other hand, condition (10) implies that $K$ is conformally Leibnizian and making use of Lemma 11, we get

$$
L_{Z} g(V, W)=\frac{2 \rho}{\Omega(K)} g(V, W), \quad \forall V, W \in \Gamma(\operatorname{An}(\Omega)) .
$$

Now, let us consider $v, w \in T_{p} U_{p}$. Last equation may be rewritten as

$$
\begin{gathered}
\lim _{\varepsilon \rightarrow 0} \frac{1}{\varepsilon}\left[g_{\Phi_{s+\varepsilon}(q)}\left(d \Phi_{s+\varepsilon}(v), d \Phi_{s+\varepsilon}(w)\right)-g_{q}\left(d \Phi_{s}(v), d \Phi_{s}(w)\right)\right] \\
=\frac{2 \rho}{\Omega(K)}\left(\Phi_{s}(q)\right) g\left(d \Phi_{s}(v), d \Phi_{s}(w)\right) .
\end{gathered}
$$

If we denote by

$$
\eta(s):=\left.\Psi^{*} g\right|_{(s, q)}((0, v),(0, w))=g\left(d \Phi_{s}(v), d \Phi_{s}(w)\right),
$$

and taking into account Lemma 11, the following differential equation holds

$$
\eta^{\prime}(s)=\frac{2 \rho}{\Omega(K)}\left(\Phi_{s}(p)\right) \eta(s) .
$$

Thus, defining the function

$$
f(s):=\exp \left(\int_{0}^{s} \frac{\rho}{\Omega(K)}\left(\Phi_{l}(p)\right) d l\right),
$$

we conclude that $\Psi^{*} g=f^{2}(t) g_{p}$, i.e., the induced metric on $I \times U_{p}$ is like (1), with $h=g_{p}$ and scale factor equal to (11).

Finally, we check that $Z$ is an irrotational and geodesic vector field. From (10),

$$
\begin{gathered}
\operatorname{Rot}(Z)(V, W)=g\left(\nabla_{V} Z, W\right)-g\left(\nabla_{W} Z, V\right) \\
=\frac{1}{\Omega(K)}\left[g\left(\nabla_{V} K, W\right)-g\left(\nabla_{W} K, V\right)\right]=0,
\end{gathered}
$$

for all $V, W \in \Gamma(\operatorname{An}(\Omega))$. By using Lemma 11, a straightforward computation gives that $\nabla_{Z} Z=0$. Since there is only one connection $\nabla$ on $M$, it must be the induced one by means of $\Psi,\left(\Psi^{-1}\right)^{*} \nabla^{G G R W}$, from the GGRW spacetime

$$
\left(I \times U_{p}, d \pi_{I},\left(f \circ \pi_{I}\right)^{2} \pi_{U_{p}}^{*} g_{p}, \nabla^{G G R W}\right) .
$$

Therefore, $\Psi$ is a Galilean diffeomorphism, and the proof is complete.

\section{Global GGRW decompositions}

We know that an ICL spacetime is locally a GGRW spacetime. Now, our aim here consists in to look for additional assumptions on the geometry of an ICL spacetime, which lead to a global splitting as a GGRW spacetime. This type of question has been yet discussed several times in the relativistic setting (see for instance, [5], [10], [11] and [1]), i.e., under what conditions on the geometry of a relativistic spacetime, this admits a global decomposition as a warped product space or, in particular, as a GRW spacetime. 
Theorem 13 A Gailean spacetime $(M, \Omega, g, \nabla)$, whose 1-form $\Omega$ is exact, admits a global decomposition as a GGRW spacetime if and only if it is an ICL spacetime with a timelike irrotational conformally vector field $K$, such that the flow of the associated field of observers, $Z:=\frac{1}{\Omega(K)} K$, is well defined and onto in a domain $I \times \mathcal{F}$ for some interval $I \subseteq \mathbb{R}$ and some leaf of the foliation $\mathcal{F}$ induced by $\Omega$.

Proof. Since $\Omega$ is exact, $\Omega=d T$ for some absolute time function $T \in C^{\infty}(M)$. Hence, the leaves of the foliation are exactly the level sets of $T, \mathcal{F}_{t}=\{q \in M: T(q)=t\}$. We fix a leaf $\mathcal{F}_{0}$ of the foliation induced by $\Omega$. Denoting by $\Phi_{s}$ the global flow of $Z$, we can build the same application of Th.12,

$$
\Psi: I \times \mathcal{F}_{0} \longrightarrow M, \quad(t, p) \longmapsto \Phi_{t}(p),
$$

that is onto by hypothesis. In order to prove the injectivity, we only need to check that each integral curve of $Z$ intersect to each leaf of $\mathcal{F}$ only once.

We proceed reasoning by contradiction, and we suppose that an integral curve of $Z, \gamma$, cut twice the same leaf $\mathcal{F}_{b}, b \in \mathbb{R}$. In that case, there are two values $s_{1}, s_{2} \in I, s_{1}<s_{2}$, such that $(T \circ \gamma)\left(s_{1}\right)=(T \circ \gamma)\left(s_{2}\right)$. Because of the real function $T \circ \gamma: I \longrightarrow \mathbb{R}$ is smooth, the Rolle Theorem applies, and there exists $s^{*} \in\left(s_{1}, s_{2}\right)$ such that

$$
\frac{d}{d s}(T \circ \gamma)\left(s^{*}\right)=0, \quad \Longleftrightarrow \quad d T\left(Z\left(\gamma\left(s^{*}\right)\right)\right)=0 .
$$

But this is a contradiction, because $Z$ is a field of observers.

Making use of the bijectivity of $\Psi$, together with the local result shown in Th.12, we conclude the proof.

Remark 14 (i) Note that the hypothesis on the absolute clock $\Omega$ automatically holds when the spacetime is simply connected. (ii) Note that the assumption on the flow of $Z$ trivially holds when $Z$ is complete.

Taking into account the previous Remark, we can assert

Corollary 15 Let $(M, \Omega, g, \nabla)$ be an ICL spacetime with timelike irrotational conformally Leibnizian vector field $K$. If the absolute clock $\Omega$ is exact and $\frac{1}{\Omega(K)} K$ is complete, then $M$ globally splits as a GGRW spacetime.

To end this work, we present a global splitting result when the spacetime is spatially compact, that is, when the leaves of the spacelike foliation are compact.

Theorem 16 Let $(M, \Omega, g, \nabla)$ be an ICL spacetime with $\Omega$ exact. If the leaves of the foliation induced by $\Omega$ are compact, then $M$ is a GGRW spacetime.

Proof. Let $\Phi: \mathcal{D} \longrightarrow M$ be the maximal local flow of the associated field of observers $Z$ and $p \in M$ a point located in a leaf $\mathcal{F}_{p}$ of the induced foliation. For each $q \in \mathcal{F}_{p}$, there exist a neighbourhood $U_{q}$ on $\mathcal{F}_{p}$ and interval $I_{p}$ such that $\Phi$ is defined in $I_{p} \times U_{p}$. Since $\mathcal{F}_{p}$ is compact, it can be written as $\mathcal{F}_{p}=\cup_{q \in A} U_{q}$, with $A$ a finite set. Taking $I=\cap_{q \in A} I_{q}$, we conclude that the flow $\Phi$ is well defined in a domain $I \times \mathcal{F}_{p}$, being $I$ an interval $(a, b)$.

Assume that $I$ is the maximal interval where $\Phi: I \times \mathcal{F} \longrightarrow M$ is defined. We are going to see that $I$ is also the maximal definition interval of each integral curve with initial value 
in $\mathcal{F}$. Suppose there exists $p_{0} \in \mathcal{F}$ such that $\Phi\left(\cdot, p_{0}\right)$ is defined in $(a, b+\epsilon)$. Since all leaves of the foliation are compact, we can take $\delta>0$ such that $(-\delta, \delta) \times \mathcal{F}_{\Phi_{\left(b, p_{0}\right)}} \subset \mathcal{D}$. Thus, it is possible to define the extension flow,

$$
\bar{\Phi}(t, q)=\left\{\begin{array}{llc}
\Phi(t, q) & \text { if } \quad t \in(a, b), \\
\Phi\left(t-b+\frac{\delta}{2}, \Phi\left(b-\frac{\delta}{2}, q\right)\right) & \text { if } \quad t \in(b-\delta, b+\delta) .
\end{array}\right.
$$

This is a contradiction.

Finally, we have to prove that $\Phi: I \times \mathcal{F} \longrightarrow M$ is onto. Consider a point $q$ in the complementary of $\Phi(I \times \mathcal{F})$ and take the maximal interval $J$ where $\Phi: J \times \mathcal{F}_{q} \longrightarrow M$ is defined. The set $\Phi(I \times \mathcal{F}) \cap \Phi\left(I \times \mathcal{F}_{q}\right)$ must be empty; if a point $q_{0}$ was in the intersection, then the integral curve of $Z$ passing through $q_{0}$ could be defined on an interval bigger than $I$. But this contradicts the maximality of $I$. Hence, taking into account that $\Phi(I \times \mathcal{F})$ and its complementary are open in $M$ and $M$ is connected, we conclude that $\Phi(I \times \mathcal{F})=M$. The same arguments of Th.13 serves to end the proof.

\section{References}

[1] J.A. Aledo, A. Romero and R.M. Rubio, The existence and uniqueness of standard static splitting, Classical Quant. Grav., 32 (2015), 105004 (1-9).

[2] L.J. Alías, A. Romero and M. Sánchez, Uniqueness of complete spacelike hypersurfaces of constant mean curvature in Generalized Robertson-Walker spacetimes, Gen. Relat. Gravit., 27 (1995), 71-84.

[3] A.N. Bernal, M. López, M. Sánchez, Fundamental units of length and time, Found. Phys., 32 (2002), 77-108.

[4] A.N. Bernal, M. Sánchez, Leibnizian, Galilean and Newtonian structures of space-time J. Math. Phys., 44 (2003), 1129-1149.

[5] M. Caballero, A. Romero and R.M. Rubio, Constant mean curvature spacelike hypersurfaces in Lorentzian manifolds with a timelike gradient conformal vector field, Class. Quantum Grav., 28 (2011), 145009-145022.

[6] A.M. Candela, A. Romero and M. Sánchez, Completeness of the trajectories of particles coupled to a general force field, Arch. Rational Mech. Anal., 208 (2013), 255-274.

[7] E. Cartan, Les variètés a conexion affine, Ann. Ec. Norm. Sup., 40 (1923), 1-25.

[8] E. Cartan, Les variètés a conexion affine (suite), Ann. Ec. Norm. Sup., 41 (1924), $325-412$.

[9] J.L Flores, M. Sánchez, Geodesic connectedness and conjugate points in GRW spacetimes, J. Geom. Phys., 36 (2000), 285-314.

[10] J.L. Flores, The Riemannian and Lorentzian splitting theorems, Atlantis Trans. Geom., Springer 1 (2017), 1-20. 
[11] M. Gutierrez and B. Olea, Global decomposition of a Lorentzian manifold as a Generalized Robertson-Walker spacetime, Diff. Geom. Appl., 27 (2009), 146-156.

[12] F. Müler-Hoissen, The cosmological principle and a generalization of Newton's theory of gravitation, Gen. Relat. Gravitation, 15 (1983), 1051-1066.

[13] B. O'Neill, Semi-Riemannian Geometry with Applications to Relativity, Pure Appl. Math., 103, Academic Press, New York, 1983.

[14] M. Sánchez, On the geometry of generalized Robertson-Walker spacetimes: geodesics Gen. Relat. Gravitation 30 (1998), 915-932.

[15] M. Sánchez, On the geometry of generalized Robertson-Walker spacetimes: curvature and Killing fields. J. Geom. Phys., 31 (1999), 1-15.

[16] F.W. Warner, Foundations of Differentiable Manifolds and Lie Groups, Grad. Texts Math., Springer-Verlag, 1983.

[17] E. Zafiris, Irreducible decomposition of Einsteins equations in spacetimes with symmetries, Ann. Phys. 263 (1998), 155-78. 